CTP-SCU/2018006

\title{
Minimal Length Effects on Chaotic Motion of Particles around Black Hole Horizon
}

\author{
Fenghua Lu.* Jun Tao $\dagger^{\dagger}$ and Peng Wang $\ddagger$ \\ Center for Theoretical Physics, College of Physical Science and Technology, \\ Sichuan University, Chengdu, 610064, PR China
}

\begin{abstract}
Recently, it was conjectured that the Lyapunov exponent of chaotic motion of a particle in a black hole is universally bounded from above by the surface gravity of the black hole. On the other hand, the minimal length appears in various theories of quantum gravity and leads to the deformed canonical position-momentum commutation relation. In this paper, we use the Hamilton-Jacobi method to study effects of the minimal length on the motion of a massive particle perturbed away from an unstable equilibrium near the black hole horizon. We find that the minimal length effects make the particle move faster away from the equilibrium, and hence the corresponding Lyapunov exponent is greater than that in the usual case with the absence of the minimal length. It therefore shows that if the minimal length effects are taken into account, the conjectured universal bound on the Lyapunov exponent could be violated.
\end{abstract}

\footnotetext{
*Electronic address: 2013322020001@stu.scu.edu.cn

${ }^{\dagger}$ Electronic address: taojun@scu.edu.cn

${ }^{\ddagger}$ Electronic address: pengw@scu.edu.cn
} 


\section{Contents}

I. Introduction

II. Rolling Solutions of Particles near Black Hole Horizon

A. Usual Case

B. Minimal Length Deformed Case

III. Discussion and Conclusion

\section{Acknowledgments}

\section{References}

\section{INTRODUCTION}

Various quantum theories of gravity such as string theory [1-4] predict the existence of a minimal measurable length. To incorporate the minimal length into quantum mechanics, the generalized uncertainty principle (GUP) [5, 6] has been proposed, in which there is a non-zero lower bound for the uncertainty in position. Moreover, the GUP can lead to the minimal length deformed fundamental commutation relation. For a 1D quantum system, the deformed commutator between position and momentum can assume the following form

$$
[X, P]=i \hbar\left(1+\beta P^{2}\right)
$$

where $\beta$ is some deformation parameter, and the minimal measurable length is $\Delta X_{\min }=$ $\hbar \sqrt{\beta}$. The deformed quantum mechanics with modification of the usual canonical commutation relations has been investigated intensively for various quantum systems, e.g. the harmonic oscillator [7], Coulomb potential [8, 9], and gravitational well [10, 11], quantum optics [12, 13] and compact stars [14, 15].

In the classical limit $\hbar \rightarrow 0$, the deformed quantum mechanical commutator is replaced by the deformed Poisson bracket for corresponding classical variables:

$$
\frac{1}{i \hbar}[\hat{A}, \hat{B}] \Rightarrow\{A, B\}
$$


via which effects of the minimal length can be investigated in the classical context. The effects of the minimal length on the observational tests of general relativity, which have been performed on Earth and in the solar system, have been considered in [16 22]. The minimal length effects on the classical system were also discussed for quantum cosmology 23, 24], classical harmonic oscillator [25], and equivalence principle [26], Newtonian potential [27] and the Schroinger-Newton equation [28].

Alternatively, the classical limit of deformed Newtonian dynamics and general relativity can be investigated using the Hamilton-Jacobi method. The deformed Hamilton-Jacobi equation is obtained from the WKB limit of the deformed quantum mechanics. In [29], we discussed the Hamilton-Jacobi method in the context of deformed 1D Newtonian mechanics. Later, we used the Hamilton-Jacobi method to investigate effects of the minimal length on the classical orbits of particles in a gravitation field, i.e. the precession of planetary orbits in the context of deformed Newtonian dynamics and the precession angle of planetary orbits, deflection angle of light, and time delay in radar propagation in the context of deformed general relativity [30]. Our result for the precession of planetary orbits in the context of deformed Newtonian dynamics agreed with that in [16], in which the deformed Poisson bracket was used. Moreover, the minimal length corrections to the Hawking temperature were also studied using the Hamilton-Jacobi method in [31 33].

Chaos in general relativity is an intriguing and important topic. Chaos is often used to study various nonlinear phenomena in nature, and one of chaotic behavior is that dynamical systems are highly sensitive to initial conditions. Examples of chaotic behavior of geodesic motion in various backgrounds were considered in [34-40]. Instead of point particles, the chaotic behavior of the ring string was studied in [41-43]. Recently, the motion of a particle near the horizon of the most general static black hole has been studied in [44, 45], in which the Lyapunov exponent for the motion restricted to the radial direction was found to be the surface gravity $\kappa$ of the black hole. It was further argued that there is a universal bound for the Lyapunov exponent of chaotic motions of particles in black holes:

$$
\lambda \leq \kappa
$$

Interestingly, this bound agrees with the bound predicted in [46] for quantum field theories. However, it showed in [47] that the bound (3) can be violated for a charged massive particle perturbed from an unstable equilibrium in some charged black hole when the equilibrium is 
not in the near-horizon region.

In this paper, we study quantum gravity effects on the bound (3) for motions of particles in a static black hole. Specifically, we use the deformed Hamilton-Jacobi equation to calculate the minimal length effects on the motion along radial direction of a massive particle perturbed away from an unstable equilibrium near the black hole horizon. We find that the corresponding Lyapunov exponent receives positive correction due to the minimal length, which violates the bound (3). For simplicity, we set $\hbar=c=k_{B}=1$ in this paper.

\section{ROLLING SOLUTIONS OF PARTICLES NEAR BLACK HOLE HORIZON}

In this section, we use Hamilton-Jacobi method to study the motion of a particle of mass $m$ in the near-horizon region of black holes. To be generic, we will consider a 4D spherically symmetric background metric of the form

$$
d s^{2}=-h(r) d t^{2}+\frac{d r^{2}}{g(r)}+r^{2}\left(d \theta^{2}+\sin ^{2} \theta d \phi^{2}\right)
$$

where $h(r)$ and $g(r)$ are assumed to have a simple zero at the event horizon $r=r_{+}$. At $r=r_{+}, h(r)$ and $g(r)$ can be Taylor expanded as

$$
\begin{aligned}
& g(r)=\gamma\left(r-r_{+}\right)+\cdots, \\
& h(r)=\eta\left(r-r_{+}\right)+\cdots .
\end{aligned}
$$

In this case, the surface gravity is given by

$$
\kappa=\frac{\sqrt{\gamma \eta}}{2}
$$

As in [44], an external potential $V(r)$ is introduced such that there is an unstable equilibrium outside the horizon for the particle. The presence of the unstable equilibrium means that any perturbation of the particle away from the equilibrium position causes it to roll down the effective potential. It was conjectured in [44] that such rolling solutions could put a universal bound on the Lyapunov exponent of chaotic motions around black holes. We assume that the potential is regular at the horizon and hence can set the zero of the potential at the horizon. So at $r=r_{+}, V(r)$ is expanded as

$$
V(r)=-v\left(r-r_{+}\right)+\cdots,
$$


where $v$ is some positive number since one needs a repulsive force to prevent the particle from falling into the black hole. As noted in [44], the external force associated with $V(r)$ could be electromagnetic or scalar force.

In the remainder of this section, we calculate the particle's rolling solution away from the unstable equilibrium near the horizon. We begin by computing the rolling solution in the

usual case. Although such solution has been obtained in [44], we here focus on applying the Hamilton-Jacobi method to find the solution. Then in the context of the minimal length deformed general relativity, the rolling solution are investigated via the Hamilton-Jacobi method.

\section{A. Usual Case}

When a relativistic particle of mass $m$ is moving under a central potential $V(r)$ in the metric (4), the corresponding Hamilton-Jacobi equation is

$$
-\frac{1}{h(r)}\left[\frac{\partial S}{\partial t}+V(r)\right]^{2}+g(r)\left(\partial_{r} S\right)^{2}+\frac{\left(\partial_{\theta} S\right)^{2}}{r^{2}}+\frac{\left(\partial_{\phi} S\right)^{2}}{r^{2} \sin ^{2} \theta}+m^{2}=0,
$$

where $S$ is the classical action. Since there are no explicit $t$-dependence in the HamiltonJacobi equation, we assume that

$$
S=-E t+W(r)+\Theta(\theta, \phi)
$$

where $E$ has the meaning of the energy. To separate the variable $\theta$ and $\phi$ from $r$, one can introduce a constant $L$ and has the equation for $\Theta(\theta, \phi)$

$$
\left(\frac{\partial \Theta}{\partial \theta}\right)^{2}+\frac{\left(\frac{\partial \Theta}{\partial \phi}\right)^{2}}{\sin ^{2} \theta}=L^{2}
$$

where $L$ represents the orbital angular momentum. For simplicity, we only consider the $L=0$ case, in which the particle moves in the radial direction. The equation for $W(r)$ then becomes

$$
\frac{E-V(x)}{\sqrt{h(r)}}=\sqrt{g(r) p_{r}^{2}+m^{2}},
$$

where we define $p_{r}=W^{\prime}(r)$. For the motion of the particle around the equilibrium with $p_{r}^{2} \ll m^{2}$, we can focus on the non-relativistic limit. In this limit, eqn. (11) reduces to

$$
\frac{g(r) \sqrt{h(r)} p_{r}^{2}}{2 m}+V_{\text {eff }}(r)=E,
$$


where one defines the effective potential for later use:

$$
V_{\text {eff }}(r)=V(r)+m \sqrt{h(r)} .
$$

The time-dependence of the motion is then obtained by the inverse Legendre transformation:

$$
t=\frac{\partial W(r)}{\partial E}
$$

which leads to

$$
\dot{r} \equiv \frac{d r}{d t}=\left(\frac{\partial p_{r}}{\partial E}\right)^{-1} .
$$

Solving eqn. (12) for $p_{r}$ in terms of $E$ and plugging it into eqn. (15), we find

$$
\frac{m \dot{r}^{2}}{2 g(r) \sqrt{h(r)}}+V_{\text {eff }}(r)=E .
$$

At the equilibrium $r=r_{0}$, one has that $E=V_{\text {eff }}\left(r_{0}\right)$ and $V_{\text {eff }}^{\prime}\left(r_{0}\right)=0$. Then around $r=r_{0}$, eqn. (16) can be expanded as

$$
\dot{\epsilon}^{2} \approx-\frac{V_{\mathrm{eff}}^{\prime \prime}\left(r_{0}\right) g\left(r_{0}\right) \sqrt{h\left(r_{0}\right)} \epsilon^{2}}{m}
$$

where $\epsilon=r-r_{0}$. Near the horizon, one can use eqns. (5) and (7) to show that the effective potential $V_{\text {eff }}(r)$ has an unstable equilibrium at $r_{0}=r_{+}+\frac{\eta m^{2}}{4 v^{2}}$. In this case, eqn. (17) then becomes

$$
\dot{\epsilon}= \pm \kappa \epsilon,
$$

where the plus sign corresponds to the rolling solution since the further the particle departs from the unstable equilibrium, the faster it moves away from the equilibrium. So the rolling solution is

$$
\epsilon(t)=A e^{\kappa t},
$$

where $A$ is the constant of integration.

To find the Lyapunov exponent of the rolling solution (19), we consider the corresponding Jacobian matrix $K$ (it is a number in our case), which describes the evolution of the tangent vectors:

$$
K=\kappa \text {. }
$$

To describes how a small change of $\epsilon(0)$ propagates to $\epsilon(t)$, we define $M(t)=\partial \epsilon(t) / \partial \epsilon(0)$, which satisfies

$$
\frac{d M(t)}{d t}=K M(t)
$$


Since $M(0)=1$, the solution of the above equation is

$$
M(t)=e^{\kappa t}
$$

The Lyapunov exponent $\lambda$ can calculated from $M(t)$ :

$$
\lambda=\lim _{t \rightarrow \infty} \frac{\ln M(t)}{t}=\kappa .
$$

Note that $\lambda>0$, which means that the difference between closely spaced initial conditions grows with evolution and hence is a signature of chaos.

\section{B. Minimal Length Deformed Case}

In three dimensions, a generalization of the deformed algebra (11) reads [6]

$$
\begin{aligned}
& {\left[X_{i}, P_{j}\right]=i \hbar\left[\left(1+\beta P^{2}\right) \delta_{i j}+\beta^{\prime} P_{i} P_{j}\right]} \\
& {\left[X_{i}, X_{j}\right]=i \hbar \frac{\left(2 \beta-\beta^{\prime}\right)+\left(2 \beta+\beta^{\prime}\right) \beta P^{2}}{1+\beta P^{2}}\left(P_{i} X_{j}-P_{j} X_{i}\right),} \\
& {\left[P_{i}, P_{j}\right]=0}
\end{aligned}
$$

where $\beta, \beta^{\prime}>0$ are two deformation parameters, and the minimal length becomes $\Delta X_{\min }=$ $\hbar \sqrt{\beta+\beta^{\prime}}$. In this paper, we consider the Brau reduction [9], where $\beta^{\prime}=2 \beta$ and the commutators taken between different components of the position $X_{i}$ vanish to the first order in $\beta$ and $\beta^{\prime}$. For this particular case, there is a very simple reduction of the form to the first order in $\beta$ :

$$
X_{i}=x_{i}, P_{i}=p_{i}\left(1+\beta p^{2}\right),
$$

where $x_{i}$ and $p_{i}$ are the conventional momentum and position operators satisfying

$$
\left[x_{i}, p_{j}\right]=i \hbar \delta_{i j},\left[x_{i}, x_{j}\right]=\left[p_{i}, p_{j}\right]=0
$$

and $p^{2}=\sum_{i} p_{i} p_{i}$

In [30], the deformed Hamilton-Jacobi equation for relativistic particles was derived by calculating the WKB limit of the corresponding deformed Klein-Gordon, Dirac and Maxwell's equations. Taking the potential $V(r)$ into account, we find that the deformed relativistic Hamilton-Jacobi equation to $\mathcal{O}(\beta)$ in the metric (4) is given by

$$
\frac{1}{h(r)}\left[\frac{\partial S}{\partial t}+V(r)\right]^{2}-\mathcal{X}(1+2 \beta \mathcal{X})=m^{2},
$$


where we define

$$
\mathcal{X}=g(r)\left(\partial_{r} S\right)^{2}+\frac{\left(\partial_{\theta} S\right)^{2}}{r^{2}}+\frac{\left(\partial_{\phi} S\right)^{2}}{r^{2} \sin ^{2} \theta}
$$

As in the usual case, we only consider the case with the particle moving along the radial direction. Therefore, separation of variables is done in the following simple manner:

$$
S=-E t+W(r)
$$

where $E$ is the energy of the particle. In the non-relativistic limit, solving the deformed Hamilton-Jacobi equation to $\mathcal{O}(\beta)$ gives

$$
p_{r}^{2} \equiv W^{\prime}(r)=\frac{2 m}{g(r)}\left(\frac{E-V_{\mathrm{eff}}(r)}{\sqrt{h(r)}}\right)\left[1-4 \beta m\left(\frac{E-V_{\mathrm{eff}}(r)}{\sqrt{h(r)}}\right)\right] .
$$

The time derivative of $r$ can be obtained by eqn. (15):

$$
\frac{m^{2} \dot{r}^{2}}{g(r) h(r)}=2 m\left(\frac{E-V_{\mathrm{eff}}(r)}{\sqrt{h(r)}}\right)\left[1+36 \beta m\left(\frac{E-V_{\mathrm{eff}}(r)}{\sqrt{h(r)}}\right)\right]
$$

Expanding the above equation around the equilibrium of $V_{\text {eff }}(r), r=r_{0}$, one has

$$
-\frac{V_{\mathrm{eff}}^{\prime \prime}\left(r_{0}\right) g\left(r_{0}\right) \sqrt{h\left(r_{0}\right)}}{m} \epsilon^{2} \approx \dot{\epsilon}^{2}\left(1-\sigma \dot{\epsilon}^{2}\right),
$$

where we define

$$
\sigma=\frac{6 \beta m^{2}}{g\left(r_{0}\right) h\left(r_{0}\right)}
$$

Near the horizon, to $\mathcal{O}(\beta)$, eqn. (32) reduces to

$$
\dot{\epsilon}= \pm \kappa \epsilon\left(1+\frac{\sigma}{2} \kappa^{2} \epsilon^{2}\right)
$$

where $\sigma=\frac{48 \beta v^{4}}{\kappa^{2} \eta^{2} m^{2}}$ in this case. We pick up the plus sign for the rolling solution, and the solution is

$$
\epsilon(t)=\epsilon_{0}(t)\left[1+\frac{\sigma \kappa^{2}}{2} \epsilon_{0}(t)\right]
$$

where $\epsilon_{0}(t)=A e^{\kappa t}$ and, higher order terms are discarded. Since $\sigma>0$, the effects of the minimal length tend to make the particle move faster away from the equilibrium.

The Jacobian matrix for the rolling solution is

$$
K=\kappa\left[1+\frac{3 \sigma}{2} \kappa^{2} \epsilon^{2}(t)\right] \text {. }
$$


So the corresponding $M(t)$ is given by

$$
M(t)=\exp \left[\kappa t+\frac{3}{4} \sigma \kappa^{2} A^{2}\left(e^{2 \kappa t}-1\right)\right] .
$$

To find the Lyapunov exponent, we consider the finite-time Lyapunov exponent $\lambda(t)$ :

$$
\lambda(t) \equiv \frac{\ln M(t)}{t}=\kappa\left[1+\frac{3 \sigma \kappa A^{2}\left(e^{2 \kappa t}-1\right)}{4 t}\right]>\kappa .
$$

If we naively take the limit of $\lambda(t)$ as $t$ approaches infinity, we find the Lyapunov exponent becomes positive infinity. However long before $t$ reach infinity, our effective approach has already broken down when contributions from higher order terms become important. Therefore, one might need to resort to full theory to calculate the Lyapunov exponent. On the other hand, we can use eqn. (38) to estimate the lower bound of the Lyapunov exponent. Actually noting that $\left(e^{2 \kappa t}-1\right) / t$ has a minimum value of $2 \kappa$ at $t=0$, one obtains that, to $\mathcal{O}(\beta)$, the Lyapunov exponent $\lambda$ is bounded from below as

$$
\lambda \gtrsim \kappa\left(1+\frac{3 \sigma \kappa^{2} A^{2}}{2}\right)
$$

We can further estimate $A$ by assuming that the perturbation of the particle at the equilibrium is due to thermal fluctuations of Hawking radiation. In this case, one finds that

$$
\frac{1}{2} m \dot{\epsilon}^{2} \sim \frac{1}{2} \frac{\kappa}{2 \pi \sqrt{\eta}\left(r-r_{0}\right)^{1 / 2}} \Rightarrow A^{2} \sim \frac{v}{\pi m^{2} \kappa \eta},
$$

where the equipartition theorem is used to estimate the kinetic energy of the particle at $r=r_{0}$. Finally, eqns. (39) and (40) gives that

$$
\lambda \gtrsim \kappa\left(1+\frac{72 \beta v^{5}}{\pi \kappa \eta^{3} m^{4}}\right)+\mathcal{O}\left(\beta^{2}\right) .
$$

\section{DISCUSSION AND CONCLUSION}

In this paper, we have used the Hamilton-Jacobi method to investigate effects of the minimal length on the rolling solution of a massive particle near the horizon of a spherically symmetric black hole. An external potential was introduced to put the particle at the unstable equilibrium outside the horizon, and the rolling solution describes the particle's departure from the equilibrium. In the framework of general relativity with the absence of the minimal length, the Lyapunov exponent $\lambda$ is just the surface gravity $\kappa$ of the black 
hole for such rolling solution. In the context of deformed general relativity, we found that the presence of the minimal length accelerates the rolling process, and the corresponding Lyapunov exponent $\lambda>\kappa$. Our results suggest that quantum effects could make the classical trajectory in black holes more chaotic.

In [46], it was conjectured that the Lyapunov exponent $\lambda$ of out-of-time-ordered correlators in thermal quantum systems with a large number of degrees of freedom is bounded by

$$
\lambda \leq 2 \pi T,
$$

where $T=\kappa / 2 \pi$ is the Hawking temperature. This bound is compatible with the universal bound for the Lyapunov exponent of chaotic motions of a single particle in black holes, which was conjectured in [44, 45]. In this paper, we showed that the bound (42) is violated for a particle moving near the black hole horizon if effects of the minimal length are considered. On the other hand, the presence of the minimal length could also modify the Hawking temperature of the black hole. In fact, it showed in [33] that the modified Hawking temperature is given by

$$
T=\left[1-\frac{\beta\left(m^{2}+4 \omega^{2}\right)}{4}\right] T_{0},
$$

where $T_{0}$ is the original Hawking temperature, and $m$ and $\omega$ are the mass and the energy of radiated particles, respectively. Said differently, effects of the minimal length decrease the Hawking temperature, which makes the violation of the bound (42) even worse.

In this paper, we investigated motion of a single particle outside the horizon, and hence our results do not necessarily mean the bound (42) conjectured in [46] is violated. However in a holographic framework, it is tempting to find how the corrections due to the minimal length in bulk changes the bound (42). Note that higher derivative corrections due to the minimal length for a scalar field theory in AdS space was analyzed and related to the conformal field theory on the boundary in [48].

\section{Acknowledgments}

We are grateful to Houwen $\mathrm{Wu}$ and Zheng Sun for useful discussions. This work is supported in part by NSFC (Grant No. 11005016, 11175039, 11875196 and 11375121). 
[1] G. Veneziano, "A Stringy Nature Needs Just Two Constants," Europhys. Lett. 2, 199 (1986). doi:10.1209/0295-5075/2/3/006

[2] D. J. Gross and P. F. Mende, "String Theory Beyond the Planck Scale," Nucl. Phys. B 303, 407 (1988). doi:10.1016/0550-3213(88)90390-2

[3] D. Amati, M. Ciafaloni and G. Veneziano, "Can Space-Time Be Probed Below the String Size?," Phys. Lett. B 216, 41 (1989). doi:10.1016/0370-2693(89)91366-X

[4] L. J. Garay, "Quantum gravity and minimum length," Int. J. Mod. Phys. A 10, 145 (1995) doi:10.1142/S0217751X95000085 gr-qc/9403008.

[5] M. Maggiore, "The Algebraic structure of the generalized uncertainty principle," Phys. Lett. B 319, 83 (1993) doi:10.1016/0370-2693(93)90785-G hep-th/9309034.

[6] A. Kempf, G. Mangano and R. B. Mann, "Hilbert space representation of the minimal length uncertainty relation," Phys. Rev. D 52, 1108 (1995) doi:10.1103/PhysRevD.52.1108 hep-th/9412167.

[7] L. N. Chang, D. Minic, N. Okamura and T. Takeuchi, "Exact solution of the harmonic oscillator in arbitrary dimensions with minimal length uncertainty relations," Phys. Rev. D 65, 125027 (2002) doi:10.1103/PhysRevD.65.125027 hep-th/0111181].

[8] R. Akhoury and Y. P. Yao, "Minimal length uncertainty relation and the hydrogen spectrum," Phys. Lett. B 572, 37 (2003) doi:10.1016/j.physletb.2003.07.084 hep-ph/0302108.

[9] F. Brau, "Minimal length uncertainty relation and hydrogen atom," J. Phys. A 32, 7691 (1999) doi10.1088/0305-4470/32/44/308 quant-ph/9905033.

[10] F. Brau and F. Buisseret, "Minimal Length Uncertainty Relation and gravitational quantum well,” Phys. Rev. D 74, 036002 (2006) doi:10.1103/PhysRevD.74.036002 hep-th/0605183.

[11] P. Pedram, K. Nozari and S. H. Taheri, "The effects of minimal length and maximal momentum on the transition rate of ultra cold neutrons in gravitational field," JHEP 1103, 093 (2011) doi:10.1007/JHEP03(2011)093 [arXiv:1103.1015 [hep-th]].

[12] I. Pikovski, M. R. Vanner, M. Aspelmeyer, M. S. Kim and C. Brukner, "Probing Planckscale physics with quantum optics," Nature Phys. 8, 393 (2012) doi:10.1038/nphys2262 arXiv:1111.1979 [quant-ph]].

[13] P. Bosso, S. Das and R. B. Mann, "Potential tests of the Generalized Uncer- 
tainty Principle in the advanced LIGO experiment," Phys. Lett. B 785, 498 (2018) doi:10.1016/j.physletb.2018.08.061 [arXiv:1804.03620 [gr-qc]].

[14] P. Wang, H. Yang and X. Zhang, "Quantum gravity effects on statistics and compact star configurations," JHEP 1008, 043 (2010) doi:10.1007/JHEP08(2010)043 arXiv:1006.5362 [hepth]].

[15] Y. C. Ong, "Generalized Uncertainty Principle, Black Holes, and White Dwarfs: A Tale of Two Infinities," JCAP 1809, no. 09, 015 (2018) doi:10.1088/1475-7516/2018/09/015 arXiv:1804.05176 [gr-qc]].

[16] S. Benczik, L. N. Chang, D. Minic, N. Okamura, S. Rayyan and T. Takeuchi, "Short distance versus long distance physicsThe Classical limit of the minimal length uncertainty relation," Phys. Rev. D 66, 026003 (2002) doi10.1103/PhysRevD.66.026003 hep-th/0204049.

[17] Z. K. Silagadze, "Quantum gravity, minimum length and Keplerian orbits," Phys. Lett. A 373, 2643 (2009) doi:10.1016/j.physleta.2009.05.053 [arXiv:0901.1258 [gr-qc]].

[18] F. Ahmadi and J. Khodagholizadeh, "Effect of GUP on the Kepler problem and a variable minimal length," Can. J. Phys. 92, 484 (2014) doi:10.1139/cjp-2013-0354 arXiv:1411.0241 [hep-th]].

[19] F. Scardigli and R. Casadio, "Gravitational tests of the Generalized Uncertainty Principle," Eur. Phys. J. C 75, no. 9, 425 (2015) doi:10.1140/epjc/s10052-015-3635-y arXiv:1407.0113 [hep-th]].

[20] A. Farag Ali, M. M. Khalil and E. C. Vagenas, "Minimal Length in quantum gravity and gravitational measurements," Europhys. Lett. 112, no. 2, 20005 (2015) doi:10.1209/02955075/112/20005 [arXiv:1510.06365 [gr-qc]].

[21] M. Khodadi, K. Nozari and A. Hajizadeh, "Some Astrophysical Aspects of a Schwarzschild Geometry Equipped with a Minimal Measurable Length,” Phys. Lett. B 770, 556 (2017) doi:10.1016/j.physletb.2017.05.016 [arXiv:1702.06357 [gr-qc]].

[22] F. Scardigli and R. Casadio, "Perihelion Precession and Generalized Uncertainty Principle," Springer Proc. Phys. 208, 149 (2018).

[23] S. Jalalzadeh, S. M. M. Rasouli and P. V. Moniz, "Quantum cosmology, minimal length and holography," Phys. Rev. D 90, no. 2, 023541 (2014) doi:10.1103/PhysRevD.90.023541 arXiv:1403.1419 [gr-qc]].

[24] G. S. Djordjevic, L. Nesic and D. Radovancevic, "Minisuperspace FLRW oscillator cosmo- 
logical model and generalized uncertainty principle," Class. Quant. Grav. 35, no. 19, 195002 (2018). doi:10.1088/1361-6382/aad849

[25] T. S. Quintela, Jr., J. C. Fabris and J. A. Nogueira, "The Harmonic Oscillator in the Classical Limit of a Minimal-Length Scenario," Braz. J. Phys. 46, no. 6, 777 (2016) doi:10.1007/s13538016-0457-9 arXiv:1510.08129 [hep-th]].

[26] V. M. Tkachuk, "Deformed Heisenberg algebra with minimal length and equivalence principle," Phys. Rev. A 86, 062112 (2012) doi:10.1103/PhysRevA.86.062112 arXiv:1301.1891 [gr-qc]].

[27] F. Scardigli, G. Lambiase and E. Vagenas, "GUP parameter from quantum corrections to the Newtonian potential," Phys. Lett. B 767, 242 (2017) doi:10.1016/j.physletb.2017.01.054 arXiv:1611.01469 [hep-th]].

[28] Q. Zhao, M. Faizal and Z. Zaz, "Short distance modification of the quantum virial theorem," Phys. Lett. B 770, 564 (2017) doi:10.1016/j.physletb.2017.01.029 [arXiv:1707.00636 [hep-th]].

[29] J. Tao, P. Wang and H. Yang, "Homogeneous Field and WKB Approximation In Deformed Quantum Mechanics with Minimal Length,” Adv. High Energy Phys. 2015, 718359 (2015) doi:10.1155/2015/718359 [arXiv:1211.5650 [hep-th]].

[30] X. Guo, P. Wang and H. Yang, "The classical limit of minimal length uncertainty relation: revisit with the Hamilton-Jacobi method," JCAP 1605, no. 05, 062 (2016) doi:10.1088/14757516/2016/05/062 arXiv:1512.03560 [gr-qc]].

[31] D. Chen, H. Wu and H. Yang, "Observing remnants by fermions' tunneling," JCAP 1403, 036 (2014) doi:10.1088/1475-7516/2014/03/036 [arXiv:1307.0172 [gr-qc]].

[32] D. Y. Chen, Q. Q. Jiang, P. Wang and H. Yang, "Remnants, fermions' tunnelling and effects of quantum gravity," JHEP 1311, 176 (2013) doi:10.1007/JHEP11(2013)176 [arXiv:1312.3781 [hep-th]].

[33] D. Chen, H. Wu, H. Yang and S. Yang, "Effects of quantum gravity on black holes," Int. J. Mod. Phys. A 29, no. 26, 1430054 (2014) doi:10.1142/S0217751X14300543 arXiv:1410.5071 [gr-qc]].

[34] Y. Sota, S. Suzuki and K. i. Maeda, "Chaos in static axisymmetric space-times. 1: Vacuum case," Class. Quant. Grav. 13, 1241 (1996) doi:10.1088/0264-9381/13/5/034 [gr-qc/9505036].

[35] W. Hanan and E. Radu, "Chaotic motion in multi-black hole spacetimes and holographic screens," Mod. Phys. Lett. A 22, 399 (2007) doi:10.1142/S0217732307022815 [gr-qc/0610119]. 
[36] J. R. Gair, C. Li and I. Mandel, "Observable Properties of Orbits in Exact Bumpy Spacetimes," Phys. Rev. D 77, 024035 (2008) doi:10.1103/PhysRevD.77.024035 arXiv:0708.0628 $[\mathrm{gr}-\mathrm{qc}]]$.

[37] V. Witzany, O. Semerák and P. Suková, "Free motion around black holes with discs or rings: between integrability and chaos - IV," Mon. Not. Roy. Astron. Soc. 451, no. 2, 1770 (2015) doi:10.1093/mnras/stv1148 [arXiv:1503.09077] [astro-ph.HE]].

[38] M. Wang, S. Chen and J. Jing, "Chaos in the motion of a test scalar particle coupling to the Einstein tensor in Schwarzschild-Melvin black hole spacetime," Eur. Phys. J. C 77, no. 4, 208 (2017) doi:10.1140/epjc/s10052-017-4792-y arXiv:1605.09506 [gr-qc]].

[39] S. Chen, M. Wang and J. Jing, "Chaotic motion of particles in the accelerating and rotating black holes spacetime," JHEP 1609, 082 (2016) doi:10.1007/JHEP09(2016)082 arXiv:1604.02785 [gr-qc]].

[40] C. Y. Liu, "Chaotic Motion of Charged Particles around a Weakly Magnetized Kerr-Newman Black Hole," arXiv:1806.09993 [gr-qc].

[41] A. V. Frolov and A. L. Larsen, "Chaotic scattering and capture of strings by black hole," Class. Quant. Grav. 16, 3717 (1999) doi:10.1088/0264-9381/16/11/316 [gr-qc/9908039].

[42] L. A. Pando Zayas and C. A. Terrero-Escalante, "Chaos in the Gauge / Gravity Correspondence," JHEP 1009, 094 (2010) doi:10.1007/JHEP09(2010)094 [arXiv:1007.0277 [hep-th]].

[43] D. Z. Ma, J. P. Wu and J. Zhang, "Chaos from the ring string in a Gauss-Bonnet black hole in AdS5 space," Phys. Rev. D 89, no. 8, 086011 (2014) doi:10.1103/PhysRevD.89.086011 arXiv:1405.3563 [hep-th]].

[44] K. Hashimoto and N. Tanahashi, "Universality in Chaos of Particle Motion near Black Hole Horizon," Phys. Rev. D 95, no. 2, 024007 (2017) doi:10.1103/PhysRevD.95.024007 arXiv:1610.06070 [hep-th]].

[45] S. Dalui, B. Ranjan Majhi and P. Mishra, "Presence of horizon makes particle motion chaotic," arXiv:1803.06527 [gr-qc].

[46] J. Maldacena, S. H. Shenker and D. Stanford, "A bound on chaos," JHEP 1608, 106 (2016) doi:10.1007/JHEP08(2016)106 arXiv:1503.01409 [hep-th]].

[47] Q. Q. Zhao, Y. Z. Li and H. Lu, "Static Equilibria of Charged Particles Around Charged Black Holes: Chaos Bound and Its Violations," arXiv:1809.04616 [gr-qc].

[48] M. Faizal, A. F. Ali and A. Nassar, "Generalized uncertainty principle as a consequence of 
the effective field theory," Phys. Lett. B 765, 238 (2017) doi:10.1016/j.physletb.2016.11.054 arXiv:1701.00341 [hep-th]]. 